\title{
Dynamics of Non-adiabatic Charged Cylindrical Gravitational Collapse
}

\author{
M. Sharif *and G. Abbas ${ }^{\dagger}$ \\ Department of Mathematics, University of the Punjab, \\ Quaid-e-Azam Campus, Lahore-54590, Pakistan.
}

\begin{abstract}
This paper is devoted to study the dynamics of gravitational collapse in the Misner and Sharp formalism. We take non-viscous heat conducting charged anisotropic fluid as a collapsing matter with cylindrical symmetry. The dynamical equations are derived and coupled with the transport equation for heat flux obtained from the MüllerIsrael-Stewart causal thermodynamic theory. We discuss the role of anisotropy, electric charge and radial heat flux over the dynamics of the collapse with the help of coupled equation.
\end{abstract}

Keywords: Gravitational collapse; Electromagnetic Field; Dynamical and transport equations.

PACS: 04.20.Cv; 04.20.Dw

\section{Introduction}

One of the most important problems in the gravitation theory and relativistic astrophysics is to understand the end state of a continual gravitational collapse. A massive star undergoes to gravitational collapse at the end of its life cycle. This happens when all the internal nuclear forces fail to supply the sufficiently high pressure to counter-balance gravity. The compact

*msharif.math@pu.edu.pk

†abbasg91@yahoo.com 
objects such as white dwarfs, neutron stars and black hole are the results of possible stages of the collapsing astronomical objects. In white dwarfs and neutron stars, gravity is neutralized by electron and neutron degeneracy pressure respectively and black hole is a complete collapsed object.

Oppenheimer and Snyder (1939) are the pioneers who studied gravitational collapse of an adiabatically flowing dust. This was idealized problem because dust is unrealistic matter and one cannot ignore the effects of pressure on the formation of spacetime singularity. A more analytic analysis was made by Misner and Sharp (1964) with perfect fluid in the inner region of a star. They formulated the dynamical equations governing adiabatic relativistic collapse. In both cases, vacuum was taken in the exterior region of a star.

The concept of non-vacuum exterior of a star was introduced by Vaidya (1951) for the radiating fluid in the interior region of the star. Goswami (2007) formulated a more realistic collapsing model by taking the radiating dust matter in the interior of a star. He remarked that bounce in the collapse is due to the dissipation. Debnath et al. (2005) explored gravitational collapse of the non-adiabatic fluid by assuming quasi-spherical Szekeres spacetime in the interior and plane symmetric Vaidya solution in the exterior region. By using the local conservation of momentum, they studied the thermodynamical behavior of the collapsing matter.

An extensive literature survey (Herrera et al. 2004 and Mitra 2006)predicts that gravitational collapse is highly dissipative process. This indicates that the effects of the dissipation must be included in the study of collapse for its better understanding. Herrera and Santos (2004) explored dynamical description of gravitational collapse by using Misner and Sharp's formulation. Matter under consideration was distributed with spherically symmetric and energy loss in the form of heat flow and radiation. Chan (2001) studied the realistic model of radiating star which undergoes dissipation in the form of radial heat flow and shear viscosity. Herrera et al. (2009) also formulated the dynamical equations by including dissipation in the form of heat flow, radiation, shear and bulk viscosity and then coupled with causal transport equations with spherical symmetry. Herrera (2006) discussed the inertia of heat and its role in the dynamics of dissipative collapse with outgoing radial heat flux by using spherical symmetry.

Most of the work available in spherical symmetry is due its simplest symmetry. To generalize the geometry of the star, people worked on gravitational collapse using the non-spherical symmetry. The existence of cylindrical and 
plane gravitational waves provides strong motivation in this regard. Herrera et al. (2005) formulated the set of equations with regularity and matching conditions for the static cylindrically symmetric distribution of matter. Sharif and Ahmad (2007) studied cylindrically symmetric gravitational collapse of two perfect fluids using the high speed approximation scheme. They investigated the emission of gravitational radiations from cylindrically symmetric gravitational collapse. Nolan (2002) investigated naked singularities in the cylinderical gravitational collapse of counter rotating dust shell. Di Prisco et al. (2009) discussed the shear free cylindrical gravitational collapse by using junction conditions. Nakao et al. (2009) studied gravitational collapse of a hollow cylinder composed of dust. Recently, Sharif and Rehmat (2010) discussed the dynamics of viscous dissipative plane symmetric gravitational collapse.

The behavior of electromagnetic field in gravitational field has been the subject of interest for many people. Thorne (1965) developed the concept of cylindrical energy and investigated that a strong magnetic field along the symmetry axis may halt the cylindrical collapse of a finite cylinder before it reached to singularity. In recent papers (Sharif and Abbas 2009, 2010a, 2010b), we have studied the effects of the electromagnetic field on the gravitational collapse by taking the homogenous, non-homogeneous and spherical model. Di Prisco et al. (2007) derived the dynamical equations for the spherically symmetric collapse by including electromagnetic field. This work has been extended by Sharif and Siddiqa (2011) for the charged plane symmetric gravitational collapse. Also, Sharif and Fatima (2011) discussed dynamics of adiabatic charged viscous cylindrical gravitational collapse.

This paper is aimed to study the dynamics of non-adiabatic charged cylindrically symmetric gravitational collapse to see the effects of charge and heat flux on the process of collapse. The plan of the paper is the following. In the next section, we describe the gravitational source and the Einstein-Maxwell field equations. Section 3 is devoted to matching conditions. We formulate the dynamical equations in section $\mathbf{4}$ and the derivation of the transport equation and their coupling with the dynamical equations are presented in section 5. The last section contains the conclusion of the paper. 


\section{Interior Matter Distribution and the Field Equations}

We take non-static cylindrically symmetric as an interior metric in the comoving coordinates in the form

$$
\begin{aligned}
& d s_{-}^{2}=-A^{2} d t^{2}+B^{2} d r^{2}+C^{2}\left(d \theta^{2}+d z^{2}\right), \\
& -\infty<t<\infty, \quad 0 \leqslant r<\infty, \quad 0 \leqslant \theta \leqslant 2 \pi, \quad-\infty<z<+\infty
\end{aligned}
$$

where $A, B$ and $C$ are functions of $t$ and $r$. Matter under consideration is anisotropic fluid which undergoes dissipation in the form of heat flux. The energy-momentum tensor for such a fluid dissipating only at diffusion approximation, i.e., $\epsilon=0$ is defined as (Herrera 2006))

$$
T_{\alpha \beta}=\left(\mu+P_{\perp}\right) V_{\alpha} V_{\beta}+P_{\perp} g_{\alpha \beta}+\left(P_{r}-P_{\perp}\right) \chi_{\alpha} \chi_{\beta}+V_{\alpha} q_{\beta}+V_{\beta} q_{\alpha}
$$

where $\mu, P_{r}, P_{\perp}, q_{\alpha}, V_{\alpha}$ and $\chi_{\alpha}$ are the energy density, the radial pressure, the tangential pressure, heat flux, the four-velocity of the fluid and the unit four-vector along the radial direction respectively. For the metric (1), the four-vector velocity, heat flux and unit four-vector along the radial direction are given by

$$
V^{\alpha}=A^{-1} \delta_{0}^{\alpha}, \quad \chi^{\alpha}=B^{-1} \delta_{1}^{\alpha}, \quad q^{\alpha}=B^{-1} q \delta_{1}^{\alpha},
$$

which satisfy

$$
V^{\alpha} V_{\alpha}=-1, \quad \chi^{\alpha} \chi_{\alpha}=1, \quad \chi^{\alpha} V_{\alpha}=0, \quad q^{\alpha} V_{\alpha}=0 .
$$

We can write the electromagnetic energy-momentum tensor in the form

$$
T_{\alpha \beta}^{(e m)}=\frac{1}{4 \pi}\left(F_{\alpha}^{\gamma} F_{\beta \gamma}-\frac{1}{4} F^{\gamma \delta} F_{\gamma \delta} g_{\alpha \beta}\right)
$$

The Maxwell equations are given by

$$
\begin{aligned}
F_{\alpha \beta} & =\phi_{\beta, \alpha}-\phi_{\alpha, \beta}, \\
F_{; \beta}^{\alpha \beta} & =4 \pi J^{\alpha},
\end{aligned}
$$

where $F_{\alpha \beta}$ is the Maxwell field tensor, $\phi_{\alpha}$ is the four potential and $J_{\alpha}$ is the four current. Since the charge is at rest with respect to the co-moving 
coordinate system, thus the magnetic field is zero. Consequently, the four potential and the four current will become

$$
\phi_{\alpha}=\phi \delta_{\alpha}^{0}, \quad J^{\alpha}=\sigma V^{\alpha},
$$

where $\phi=\phi(t, r)$ is an arbitrary function and $\sigma=\sigma(t, r)$ is the charge density.

For the interior spacetime, using Eq.(6), the Maxwell field equations take the following form

$$
\begin{aligned}
\phi^{\prime \prime}-\left(\frac{A^{\prime}}{A}+\frac{B^{\prime}}{B}-2 \frac{C^{\prime}}{C}\right) \phi^{\prime} & =4 \pi \sigma A B^{2}, \\
\dot{\phi}^{\prime}-\left(\frac{\dot{A}}{A}+\frac{\dot{B}}{B}-2 \frac{\dot{C}}{C}\right) \phi^{\prime} & =0
\end{aligned}
$$

where dot and prime represent the partial derivatives with respect to $t$ and $r$ respectively. Integration of Eq.(7) implies that

$$
\phi^{\prime}=\frac{2 s A B}{C^{2}},
$$

where $s(r)=2 \pi \int_{0}^{r} \sigma B C^{2} d r$ is the total charge distributed per unit length of the cylinder and is the consequence of law of conservation of charge, $J_{; \mu}^{\mu}=0$. Obviously Eq.(8) is identically satisfied by Eq.(91).

The Einstein field equations, $G_{\alpha \beta}=8 \pi\left(T_{\alpha \beta}+T_{\alpha \beta}^{(e m)}\right)$, for the metric (1) can be written as

$$
\begin{aligned}
8 \pi\left(T_{00}+T_{00}^{(e m)}\right) & =8 \pi \mu A^{2}+\frac{4 s^{2} A^{2}}{C^{4}} \\
& =\frac{\dot{C}}{C}\left(2 \frac{\dot{B}}{B}+\frac{\dot{C}}{C}\right)+\left(\frac{A}{B}\right)^{2}\left(-2 \frac{C^{\prime \prime}}{C}+2 \frac{B^{\prime} C^{\prime}}{B C}-\left(\frac{C^{\prime}}{C}\right)^{2}\right) \\
8 \pi\left(T_{01}+T_{01}^{(e m)}\right) & =8 \pi q=\frac{2}{A B}\left(\frac{\dot{C}^{\prime}}{C}-\frac{\dot{B} C^{\prime}}{B C}-\frac{\dot{C} A^{\prime}}{C A}\right), \\
8 \pi\left(T_{11}+T_{11}^{(e m)}\right) & =8 \pi P_{r} B^{2}-\frac{4 s^{2} B^{2}}{C^{4}} \\
& =-\left(\frac{B}{A}\right)^{2}\left(2 \frac{\ddot{C}}{C}+\left(\frac{\dot{C}}{C}\right)^{2}-2 \frac{\dot{A} \dot{C}}{A C}\right)+\left(\frac{C^{\prime}}{C}\right)^{2}+2 \frac{A^{\prime} C^{\prime}}{A C}
\end{aligned}
$$




$$
\begin{aligned}
8 \pi\left(T_{22}+E_{22}\right) & =8 \pi P_{\perp} C^{2}+\frac{4 s^{2}}{C^{2}} \\
& =-\left(\frac{C}{A}\right)^{2}\left(\frac{\ddot{B}}{B}+\frac{\ddot{C}}{C}-\frac{\dot{A}}{A}\left(\frac{\dot{B}}{B}+\frac{\dot{C}}{C}\right)+\frac{\dot{B} \dot{C}}{B C}\right) \\
& +\left(\frac{C}{B}\right)^{2}\left(\frac{A^{\prime \prime}}{A}+\frac{C^{\prime \prime}}{C}-\frac{A^{\prime}}{A}\left(\frac{B^{\prime}}{B}-\frac{C^{\prime}}{C}\right)-\frac{B^{\prime} C^{\prime}}{B C}\right)
\end{aligned}
$$

The C-energy for the cylindrically symmetric spacetime is defined by (Thorne 1965)

$$
E=\frac{1}{8}\left(1-l^{-2} \nabla^{a} \tilde{r} \nabla_{a} \tilde{r}\right)
$$

The circumference radius $\rho$, specific length $l$ and areal radius $\tilde{r}$ can be defined as

$$
\rho^{2}=\xi_{(1) a} \xi_{(1)}^{a}, \quad l^{2}=\xi_{(2) a} \xi_{(2)}^{a}, \quad \tilde{r}=\rho l
$$

where $\xi_{(1)}=\frac{\partial}{\partial \theta}, \xi_{(2)}=\frac{\partial}{\partial z}$ are Killing vectors and $E$ represents the gravitational energy per unit specific length of the cylinder.

The specific energy of the cylinder (Poisson 2004) analogous to Misner and Sharp energy for the spherical symmetry in the interior region with the contribution of electromagnetic field can be written as follows

$$
E^{\prime}=\frac{l}{8}+\frac{C}{2}\left(\frac{\dot{C}^{2}}{A^{2}}-\frac{C^{2}}{B^{2}}\right)+\frac{s^{2}}{2 C}
$$

We would like to mention here that this energy is also analogous to Taub's mass function in the plane symmetric spacetime (Sharif and Rehmat 2010 ).

\section{Junction Conditions}

In this section, we assume that the $3 D$ timelike boundary surface $\Sigma$ splits the two $4 D$ cylindrically symmetric spacetimes $V^{+}$and $V^{-}$. The metric which describes the internal region $V^{-}$is given by Eq.(1) while for the representation of exterior region $V^{+}$, a metric in the retarded time coordinate is considered. If $M(\nu)$ and $Q(\nu)$ are mass and charge, respectively, in retarded time then the corresponding cylindrically symmetric spacetime given 
by (Chao-Guang 1995) will take the form

$$
d s_{+}^{2}=-\left(\frac{-2 M(\nu)}{R}+\frac{Q^{2}(\nu)}{R^{2}}\right) d \nu^{2}-2 d R d \nu+R^{2}\left(d \theta^{2}+d z^{2}\right),
$$

where $\nu$ is the retarded time coordinate. We can write the induced metric for the hypersurface $\Sigma$ in the following form

$$
\left(d s^{2}\right)_{\Sigma}=-d \tau^{2}+y^{2}(\tau)\left(d \theta^{2}+d z^{2}\right),
$$

where $\xi^{i} \equiv(\tau, \phi, z)(i=0,2,3)$ represent the intrinsic coordinates of $\Sigma$.

The Darmois junction conditions (Darmois, 1927) can be stated as follows:

- The continuity of the first fundamental form over the hypersurface $\Sigma$ i.e.,

$$
\left(d s^{2}\right)_{\Sigma}=\left(d s_{-}^{2}\right)_{\Sigma}=\left(d s_{+}^{2}\right)_{\Sigma}
$$

- The continuity of the second fundamental form over the hypersurface $\Sigma$

$$
\left[K_{i j}\right]=K_{i j}^{+}-K_{i j}^{-}=0
$$

Here, $K_{i j}^{ \pm}$is the extrinsic curvature given by

$$
K_{i j}^{ \pm}=-n_{\sigma}^{ \pm}\left(\frac{\partial^{2} \chi_{ \pm}^{\sigma}}{\partial \xi^{i} \partial \xi^{j}}+\Gamma_{\mu \nu}^{\sigma} \frac{\partial \chi_{ \pm}^{\mu} \partial \chi_{ \pm}^{\nu}}{\partial \xi^{i} \partial \xi^{j}}\right), \quad(\sigma, \mu, \nu=0,1,2,3) .
$$

where $n_{\sigma}^{ \pm}$are the components of outward unit normal to the hypersurface in the coordinates $\chi^{ \pm \mu}$.

We can write the equations of hypersurface as follows

$$
\begin{aligned}
h_{-}(t, r) & =r-r_{\Sigma}=0, \\
h_{+}(\nu, R) & =R-R_{\Sigma}(\nu)=0,
\end{aligned}
$$

where $r_{\Sigma}$ is a constant. Using above equations, we have the interior and exterior spacetimes on $\Sigma$ as follows

$$
\begin{aligned}
\left(d s_{-}^{2}\right)_{\Sigma} & =-A^{2}\left(t, r_{\Sigma}\right) d t^{2}+C^{2}\left(t, r_{\Sigma}\right)\left(d \theta^{2}+d R_{\Sigma}(\nu) 2\right) . \\
\left(d s_{+}^{2}\right)_{\Sigma} & =-\left[\left(\frac{-2 M(\nu)}{R_{\Sigma}(\nu)}+\frac{Q^{2}(\nu)}{R_{\Sigma}(\nu)^{2}}\right)+\frac{2 d R_{\Sigma}(\nu)}{d \nu}\right] d \nu^{2} \\
& +R_{\Sigma}^{2}\left(d \theta^{2}+d z^{2}\right) .
\end{aligned}
$$


The continuity of the first fundamental form gives

$$
\begin{aligned}
R_{\Sigma}(\nu) & =C\left(t, r_{\Sigma}\right) \\
\frac{d t}{d \tau} & =\frac{1}{A} \\
\frac{d \nu}{d \tau} & =\left[\left(\frac{-2 M(\nu)}{R_{\Sigma}}+\frac{Q^{2}(\nu)}{R_{\Sigma}^{2}}\right)+\frac{2 d R_{\Sigma}}{d \nu}\right]^{\frac{-1}{2}}
\end{aligned}
$$

Now we consider the second fundamental form over $\Sigma$. For this purpose, we need the outward unit normals to $\Sigma$ using Eqs.(21) and (22)

$$
\begin{aligned}
& n_{a}^{-}=B(0,1,0,0), \\
& n_{a}^{+}=\left(\frac{-2 M(\nu)}{R_{\Sigma}}+\frac{Q^{2}(\nu)}{R_{\Sigma}^{2}}+\frac{2 d R_{\Sigma}}{d \nu}\right)^{\frac{-1}{2}}\left(-\frac{d R_{\Sigma}}{d \nu}, 1,0,0\right) .
\end{aligned}
$$

The non-zero components of the extrinsic curvature $K_{i j}^{ \pm}$are

$$
\begin{aligned}
K_{00}^{-} & =-\left(\frac{A^{\prime}}{A B}\right)_{\Sigma}, \\
K_{00}^{+} & =\left[\frac{d^{2} \nu}{d \tau^{2}}\left(\frac{d \nu}{d \tau}\right)^{-1}-\left(\frac{M}{R^{2}}-\frac{Q^{2}}{R^{3}}\right)\left(\frac{d \nu}{d \tau}\right)\right]_{\Sigma} . \\
K_{22}^{-} & =K_{33}^{-}=\left(\frac{C C^{\prime}}{B}\right)_{\Sigma}, \\
K_{22}^{+} & =\left[R \frac{d R}{d \tau}+\left(\frac{Q^{2}}{R}-2 M\right) \frac{d \nu}{d \tau}\right]_{\Sigma}=K_{33}^{+} .
\end{aligned}
$$

The continuity of the extrinsic curvature components yields

$$
\begin{array}{r}
{\left[\frac{d^{2} \nu}{d \tau^{2}}\left(\frac{d \nu}{d \tau}\right)^{-1}-\left(\frac{M}{R^{2}}-\frac{Q^{2}}{R^{3}}\right)\left(\frac{d \nu}{d \tau}\right)\right]_{\Sigma}=-\left(\frac{A^{\prime}}{A B}\right)_{\Sigma}} \\
{\left[R \frac{d R}{d \tau}+\left(\frac{Q^{2}}{R}-2 M\right) \frac{d \nu}{d \tau}\right]_{\Sigma}=\left(\frac{C C^{\prime}}{B}\right)_{\Sigma}}
\end{array}
$$

Using Eqs.(25)-(27), (10) and (11) in Eqs.(134) and (35), it follows that

$$
\begin{array}{r}
E^{\prime}-M \stackrel{\Sigma}{=} \frac{l}{8} \Leftrightarrow s \stackrel{\Sigma}{=} Q, \\
q \stackrel{\Sigma}{=} P_{r}-\frac{3 s^{2}}{2 C^{4}} .
\end{array}
$$


The first equation indicates that the difference between two masses is equal to $\frac{l}{8}$ as shown in the adiabatic case (Sharif and Fatima 2011). This is due to the least unsatisfactory definition of Thorne C-energy (Thorne 1965). The second equation describes a relation between heat flux, radial pressure and charge over the hypersurface $\Sigma$. It is obvious from this equation that for uncharged radiating fluid, radial pressure and heat flux are equal over the boundary of the collapsing cylinder.

\section{The Dynamical Equations}

Here we derive the dynamical equations for non-adiabatic charged anisotropic fluid. The energy-momentum conservation, $\left(T^{\alpha \beta}+T^{(e m) \alpha \beta}\right)_{; \beta}=0$, implies that

$$
\begin{aligned}
\left(T^{\alpha \beta}+T^{(e m)^{\alpha \beta}}\right)_{; \beta} V_{\alpha}= & -\frac{\dot{\mu}}{A}-\frac{\dot{B}}{A B}\left(\mu+P_{r}\right)-\frac{2 \dot{C}}{A C}\left(\mu+P_{\perp}\right) \\
& -\frac{2 q}{B}\left(\frac{A^{\prime}}{A}+\frac{C^{\prime}}{C}\right)-\frac{q^{\prime}}{B}=0
\end{aligned}
$$

and

$$
\begin{aligned}
\left(T^{\alpha \beta}+T^{(e m)^{\alpha \beta}}\right)_{; \beta} \chi_{a} & =\frac{1}{B} P_{r}^{\prime}+\frac{A^{\prime}}{A B}\left(\mu+P_{r}\right)+\frac{\dot{q}}{A}+\frac{2 q}{A}\left(\frac{\dot{B}}{B}+\frac{\dot{C}}{C}\right) \\
& +\frac{2 C^{\prime}}{B C}\left(P_{r}-P_{\perp}\right)-\frac{s s^{\prime}}{\pi B C^{4}}=0
\end{aligned}
$$

Following Misner and Sharp formalism (Misner and Sharp 1964), we discuss the dynamics of the collapsing system. We introduce proper time derivative as well as the proper radial derivative constructed from the circumference radius of a cylinder inside $\Sigma$ as (Sharif and Fatima 2011)

$$
D_{T}=\frac{1}{A} \frac{\partial}{\partial t}, \quad D_{R}=\frac{1}{R^{\prime}} \frac{\partial}{\partial r}, \quad R=C .
$$

The fluid velocity in the case of collapse can be defined as

$$
U=D_{T}(R)=D_{T}(C)<0 .
$$

Consequently, we can write Eq.(15) as

$$
\tilde{E}=\frac{C^{\prime}}{B}=\left[U^{2}+\frac{s^{2}}{C^{2}}-\frac{2}{C}\left(E^{\prime}-\frac{1}{8}\right)\right]^{1 / 2} .
$$


Using Eqs.(11), (12), (15) and (40), the time rate of change of the C-energy turns out to be

$$
D_{T} E^{\prime}=-4 \pi R^{2}\left[\left(P_{r}-\frac{1}{32 \pi R^{2}}\right) U+q \tilde{E}\right]+\frac{3 s^{2} U}{2 R^{2}} .
$$

This equation represents the variation of total energy inside the collapsing cylinder. Since $U<0$, the first term on the right hand side of this equation will increase the energy of the system provided that the factor within the round brackets is positive. The second term in the square brackets due to negative sign describes the outflow of energy in the form of radiation during the collapse. For the collapsing cylinder containing the same species of the charges, the third term will decrease the energy of the system as $\frac{3 s^{2}}{2 R^{2}}$ plays the role of Coulomb repulsive force and $U<0$.

Similarly, using Eqs.(10), (11), (15) and (40), we obtain

$$
D_{R} E^{\prime}=4 \pi R^{2}\left(\mu+q \frac{U}{\tilde{E}}\right)+\frac{l}{8}+\frac{s}{R} D_{R} s+\frac{3 s^{2}}{2 R^{2}} .
$$

This equation gives the variation of energy between the adjacent cylindrical surfaces inside the matter distribution. The first term is the energy density of the fluid element along with heat flux contribution. Since $U<0$, the heat flux factor decreases the energy of the system during the collapse of cylinder. The term $\frac{l}{8}$ comes from the definition of C-energy and the remaining terms are due to the electromagnetic field.

Using Eqs.(12), (15), (41) and (42), we can obtain the acceleration $D_{T} U$ of a collapsing matter inside $\Sigma$

$$
D_{T} U=-\frac{1}{R^{2}}\left(E^{\prime}-\frac{l}{8}\right)-4 \pi R P_{r}+\frac{\tilde{E} A^{\prime}}{A B}+\frac{5 s^{2}}{2 R^{3}} .
$$

Inserting the value of $\frac{A^{\prime}}{A}$ from Eq.(45) into Eq.(39), it follows that

$$
\begin{aligned}
\left(\mu+P_{r}\right) D_{T} U= & -\left(\mu+P_{r}\right)\left[\frac{1}{R^{2}}\left(E^{\prime}-\frac{l}{8}\right)+4 \pi P_{r} R-\frac{5 s^{2}}{2 R^{3}}\right] \\
& -\tilde{E}\left[D_{T} q+\frac{4 q U}{R}+2 q G\right] \\
& -\tilde{E}^{2}\left[D_{R} P_{r}+2\left(P_{r}-P_{\perp}\right) \frac{1}{R}-\frac{s}{\pi R^{4}} D_{R} s\right]
\end{aligned}
$$


where $G=\frac{1}{A}\left(\frac{\dot{B}}{B}-\frac{\dot{C}}{C}\right) \neq 0$ for simplicity. Now the complete dynamics of the system is described by Eq.(46). The system will evolve radially outward or inward according to $D_{T} U<0$ or $D_{T} U>0$. Thus the terms in Eq.(46) contributing negatively, favors the collapse while the other contribution prevents the collapse. If both of these cancel each other then there will be a hydrostatic equilibrium. Since the left hand side of Eq.(46) represents force, so the factor $\mu+P_{r}$ refers to an inertial mass density independent of charge and heat flux contributions. The first and third terms on the right hand side represents the gravitational force. The second term represents the heat flux contribution which seems to leave the system (due to negative sign) through the outward radially directed streamlines. Being in the same direction of pressure, it supports the pressure and would prevent the collapse.

The term $\left(\mu+P_{r}\right)\left[\frac{1}{R^{2}}\left(E^{\prime}-\frac{l}{8}\right)+4 \pi P_{r} R-\frac{5 s^{2}}{2 R^{3}}\right]$ represents the gravitational force. The factor within the first square brackets shows the effects of specific length and the electric charge on the active gravitational mass term $\left(\mu+P_{r}\right)$. The third term has three main contributions, i.e., the first is the pressure gradient which is negative, the second is the local anisotropy of the fluid which will be negative for $P_{r}<P_{\perp}$ and the third is the electromagnetic field term. For an isotropic pressure, the second contribution will be vanished. Further, following Di Prisco et al. (2007), it can be found that the third term contributes negatively for $\frac{s}{R}>D_{R} s$. Thus the third square brackets, under these conditions with negative sign, contributes positively by reducing an attractive behavior of force appearing on left hand side of the equation. Since the attractive force is decreased, so the third term prevents the gravitational collapse of the cylinder.

\section{The Transport Equation}

The transport equation predicts the processes of mass, heat and momentum transfer during the dynamics of a realistic matter. The transport equation for heat flux derived from the Müller-Israel-Stewart causal thermodynamic theory is given by (Herrera 2006)

$$
\tau h^{a b} V^{c} q_{b ; c}+q^{a}=-\kappa h^{a b}\left(T_{, b}+a_{b} T\right)-\frac{1}{2} \kappa T^{2}\left(\frac{\tau V^{b}}{\kappa T^{2}}\right)_{; b} q^{a},
$$

where $h^{a b}=g^{a b}+V^{a} V^{b}$ is the projection tensor, $\kappa$ denotes thermal conductivity, $T$ is temperature, $\tau$ stands for relaxation time which is the time 
taken by a perturbed system to return into an equilibrium state and $a_{b} T$ is the Tolman inertial term. Due to symmetry of the spacetime, the transport equation reduces to the following form

$$
\tau \dot{q}=-\frac{1}{2} \kappa q T^{2}\left(\frac{\tau}{\kappa T^{2}}\right)^{\cdot}-\frac{1}{2} \tau q\left(\frac{\dot{B}}{B}+2 \frac{\dot{C}}{C}\right)-\frac{\kappa}{B}(T A)^{\prime}-q A .
$$

Using Eqs.(40) and (41) in this equation, it follows that

$$
\begin{aligned}
D_{T} q= & -\frac{\kappa T^{2} q}{2 \tau} D_{T}\left(\frac{\tau}{\kappa T^{2}}\right)-q\left[\frac{3 U}{2 R}+G+\frac{1}{\tau}\right]-\frac{\kappa \tilde{E}}{\tau} D_{R} T-\frac{\kappa T}{\tau \tilde{E}} \\
& \times D_{T} U-\frac{\kappa T}{\tau \tilde{E}}\left[E^{\prime}+\frac{l}{8}+4 \pi P_{r} R^{3}-\frac{5 s^{2}}{2 R}\right] \frac{1}{R^{2}} .
\end{aligned}
$$

In order to understand the effects of heat flux or dissipation on collapsing process, we couple Eq.(49) with dynamical Eq.(46). Thus the replacement of Eq.(49) in Eq.(46), yields

$$
\begin{aligned}
\left(\mu+P_{r}\right)(1-\alpha) D_{T} U & =(1-\alpha) F_{\text {grav }}+F_{\text {hyd }}+\frac{\kappa \tilde{E}^{2}}{\tau} D_{R} T \\
& +\tilde{E}\left[\frac{\kappa T^{2} q}{2 \tau} D_{T}\left(\frac{\tau}{\kappa T^{2}}\right)\right]-\tilde{E} q\left(\frac{5 U}{2 R}+G-\frac{1}{\tau}\right),
\end{aligned}
$$

where $F_{\text {grav }}, F_{\text {hyd }}$ and $\alpha$ are given by the following equations

$$
\begin{aligned}
F_{\text {grav }} & =-\left(\mu+P_{r}\right)\left[E^{\prime}-\frac{C}{8}+4 \pi P_{r} R^{3}-\frac{5 s^{2}}{2 R}\right] \frac{1}{R^{2}} \\
F_{h y d} & =-\tilde{E}^{2}\left[D_{R}\left(P_{r}\right)+\frac{2}{R}\left(P_{r}-P_{\perp}\right)-\frac{\mu_{0}^{2} s D_{R} s}{4 \pi R^{4}}\right] \\
\alpha & =\frac{\kappa T}{\tau}\left(\mu+P_{r}\right)^{-1} .
\end{aligned}
$$

For the physical interpretation of Eq.(150), it can be observed that the left hand side of this equation being the product of inertial mass density $(\mu+$ $\left.P_{r}\right)(1-\alpha)$ and acceleration, $D_{T} U$ represents the Newtonian force. Thus we can write $F=\left(\mu+P_{r}\right)(1-\alpha) D_{T} U$. It is clear that when $\alpha \rightarrow 1$, then $F \rightarrow 0$, which means that there is no inertial force and matter would experience the gravitational attraction which causes the collapse. For $0<\alpha<1$, the inertial mass density goes on decreasing while $1<\alpha$ indicates the increase of inertial mass density. Of course, by the equivalence principle, there would 
occur decrease and increase in the gravitational mass. In this way, one can explicitly distinguish the expanding and collapsing mechanism during the dynamics of dissipative system.

Also, Eq.(50) implies that gravitational force is affected by the same factor but hydrodynamical force is independent of this factor. Further, as long as $\left(\mu+P_{r}\right)(1-\alpha) D_{T} U<0$, the total Newtonian force of the system remains directed downward which is the indication for the gravitational collapse and the converse is true for the expansion. If there is a continuous change in $\alpha$ from a value greater than one to less than one and vice versa, then there is a transition phase in the system and bouncing would occur. This phenomenon causes the loss of energy from the system and hence the collapsing cylinder with non-adiabatic source leads to the emission of the gravitational radiations. On the basis of this fact, the exterior of the collapsing cylinder is radiation zone which is completely described by a spacetime in radiation coordinates like in the present case.

\section{Outlook}

This paper deals with the effects of the charge and heat conduction on the dynamics of cylindrical anisotropic fluid collapse. We have extended the recent work of Sharif and Fatima (2011) to non-adiabatic case for the transportation process of heat flux during the dynamics of realistic matter collapse. For this purpose, the non-viscous heat conducting anisotropic fluid with cylindrical symmetry has been taken as the source of gravitation in the presence of electromagnetic field. Using the Misner and Sharp formalism, the dynamical equations are derived. We have found that during the collapse of non-viscous heat conducting charged anisotropic fluid, the radial heat flux and electric charge causes to reduce the energy of the system.

For $q>0, E^{\prime}=\frac{C^{\prime}}{B}>0$, Eq.(44) yields that the second term in the first square brackets is negative which indicates that heat is emitting from the system. Thus the external region of such a collapsing system, being nonvacuum, is defined by a cylindrical geometry in the radiation (single null) coordinate. This prediction is analogous to the Vaidya (1951) for spherically symmetric case. The time evolution of the system, given by dynamical equation (46), indicates that the inertial mass density is independent of heat flux and electric charge. The left hand side of this equation corresponds to the Newtonian force of the system which is decreased by the heat flux. 
Since the collapse of a star is an irreversible process. The transport process of such non-equilibrium objects and connection between their dynamics and thermodynamics are important for the better understanding of the problem. Thus using the Müller-Israel-Stewart causal thermodynamic theory, the transport equation for the dissipative fluid has been formulated and coupled to the dynamical equation. The coupled equation helps to determine the influence of the heat flux over the dynamics of collapsing cylinder. It has been found that in the coupled dynamical Eq.(501), the inertial as well as gravitational masses are influenced by the factor $(1-\alpha)$. The role of $\alpha$ during the dynamics of system can be explained as follows: For $\alpha$ tends to one, we get zero mass density. For $0<\alpha<1$, the inertial and the gravitational mass density are decreased while for $\alpha>1$, the gravitational force term becomes negative. This is the case for the reversal of collapse. The conditions on $\alpha$ have been investigated for the bouncing behavior of the system.

\section{Acknowledgment}

We would like to thank the Higher Education Commission, Islamabad, Pakistan for its financial support through the Indigenous Ph.D. 5000 Fellowship Program Batch-IV.

\section{References}

Chao-Guang, H.: Acta Phys. Sin. 4, 617(1995)

Chan, R.: Astron. Astrophys. 368, 325(2001)

Darmois, G.: Memorial des Sciences Mathematiques (Gautheir-Villars, Paris, 1927) Fasc. 25

Debnath, U., Nath, S., Chakraborty, S.: Gen. Relativ. Grav. 37, 215(2005)

Di Prisco, A., Herrera, L., Denmat, G.Le., MacCallum, M.A.H., Santos, N.O.: Phys. Rev. D76, 064017(2007)

Di Prisco, A., Herrera, L., Denmat, G.Le., MacCallum, M.A.H., Santos, N.O.: Phys. Rev. D80, 064031(2009)

Goswami, R.: Gravitational Collapse of Dustlike Matter with Heat Flux, ArXiv: 0707.1122.

Hayward, S.A.: Class. Quantum Grav.: 17, 1749(2000)

Herrera, L., Di Prisco, A., Martin, J. Ospino, J. Santos, N.O., Troconis, O.: Phys. Rev. D69, 084026(2004) 
Herrera, L., Santos, N.O.: Phys. Rev. D70, 084004(2004)

Herrera, L., Di Prisco, A., Fuenmayor, E., Troconis, O.: Int. J. Mod. Phys. D18, 129(2009)

Herrera, L.: Int. J. Mod. Phys. D15, 2197(2006)

Herrera, L., Denmat, G.Le., Marcilhacy, G., Santos, N.O.: Int. J. Mod. Phys. D14, 657(2005)

Misner, C.W., Sharp, D.: Phys. Rev. 136, B571(1964)

Mitra, A.: Phys. Rev. D74, 024010(2006)

Nolan, B.C.: Phys. Rev. D65, 104006(2002)

Nakao, K., Harada, T., Kurita, Y., Morisawa, Y.: Prog. Theor. Phys. 122, 521(2009)

Oppenheimer, J.R., Snyder, H.: Phys. Rev. 56, 455(1939)

Poisson, E.: A Relaivistic's Toolkit (Cambrridge University Press, 2004)

Sharif, M., Abbas, G.: Mod. Phys. Lett. A24, 2551(2009)

Sharif, M., Abbas, G.: J. Korean Phys. Society 56, 529(2010)

Sharif, M., Abbas, G.: Astrophys. Space Sci. 327, 285(2010)

Sharif, M., Siddiqa, A.: Gen. Relativ. Grav. 43, 37(2011)

Sharif, M., Fatima, S.: Gen. Relativ. Grav. 43, 127(2011)

Sharif, M., Ahmad, Z.: Gen. Relativ. Grav. 39, 1331(2007)

Sharif, M., Rehmat, Z.: Gen. Relativ. Grav. 42, 1795(2010)

Thorne, K.S.: Phys. Rev. 138(1965)B251; ibid. 139, B244(1965)

Vaidya, P.C.: Proc. Indian Acad. Sci. A33, 264(1951) 